\title{
PROBABILISTIC STRUCTURE OF TWO-LANE ROAD TRAFFIC
}

\author{
CARIOS F. DAGANZO \\ Department of Civil Engineering. The University of Michigan. Ann Arbor, MI 48104, U.S.A.
}

(Received 22 August 1974: in revised form 24 March 1975)

\begin{abstract}
In most predictive models for two-lane road traffic, it is assumed that platoons have no physical dimensions, thus restricting their applicability to light traffic where a platoon cannot be long enough to block the progression of the next one.

In this paper a model that can be used for heavy traffic is presented. A queueing theory approach in which vehicles are allowed to have physical dimensions yields the platoon length distribution, the delays to fast vehicles. the headway process and the flow density diagram for both the space and time processes. Unlike in otber models. the passing rate is determined from the sight distance characteristics of the road and the opposing traffic flow with an approach similar to the one used for vehicular delays at unsignalized intersections; this improves time predictive capabilities of the model.
\end{abstract}

\section{INTRODUCTION}

Since the headway process of a traffic stream on a two-lane road is a main input into such problems as pedestrian delay and vehicle crossing capacity, it is clearly of capital importance to have adequate models to describe this process appropriately. Not to be neglected are such other important characteristics of traffic as speeds, delay, and platoon length which atso affect the performance of a roadway and its side roads.

It is the purpose of this paper to present a model for traffic description that can predict the headway process, bunching, speeds, etc., on two-lane roads as a function of demand and road conditions.

The problem of predicting bunching and delays on a two-lane road is one of the most classical applications of the theory of stochastic processes to traffic problems. The topic has been widely studied; Newell (1955) introduced the first integral equation as an approach to this problem. Erlander (1967) used Gustavsson's (1967) results for passing and established an integral equation following Carlesson's (1957) approach. Newell (1966) considered interactions between two vehicles only. All of the above mentioned models assume that each vehicle has its own desired speed and is delayed by slower vehicles. However. they do not include queueing effects, and consequently, their results, when available, may be used for very light traffic only.

To avoid this unrealistic feature Andrews (1970) attempted to introduce interactions between three cars but the derivations became very involved.

Miller (1962) and Gordon and Newell (1964) included queueing in their models, and made similar assumptions regarding the passing behavior and the platoon formation mechanism: the only difference being that Miller considered a continuous range of desired speeds while Gordon and Newell considered the discrete case. Their formulations seem to be realistic but the equations derived are intractable except for the case of two speeds.

Using the same type of model, with two speeds only, Brill (1972) looked at the structure of the headway process, and Galin and Epstein (1974) considered a peculiar passing mechanism.

So far, models restricting speeds to two possible values have been the only ones to yield fairly simple solutions. However, even these models neglect the physical distances between vehicles in a platoon and hence do not apply for medium and heavy traffic because the possibility of a platoon being so long that it obstructs the progress of the next one displacing it backwards is ignored.

Both Oiver (1961) and Tanner (1961a) took into account the physical dimensions of the vehicles, and obtained the platoon length distribution. However, all vehicles were restricted to travel at the same speed.

In this paper we follow the approach of Gordon and Newell with two vehicle types, for light traffic, and generalize the results of both; Oliver-Tanner and Gordon-Newell for the case of heavy traffic by allowing the vehicles to have physical dimensions and two different desired speeds.

\section{THE LIGHT TRAFFIC MODEL}

We consider one lane of a homogeneous, infinitely long road with traffic consisting of two vehicle types with different desired speeds. The fast vehicles catch up with the slower ones and are forced to queue behind them unti] both a gap of sufficient size appears in the opposing stream and sight distance conditions are favorable. It is also assumed that only one of the vehicles in a moving queue is ready to pass, although that vehicle may not be the first one to have arrived. Using queueing theory terminology, a slow vehicle in the traffic stream performs the function of a single server and a fast one that of a customer but the queue discipline is not necessarily first come, first served.

It is assumed that the service times are negative exponentially distributed random variables with mean $\left(\mu^{-1}\right)$ and that the service mechanisms of all servers are identical and independent. These assumptions are essential to the derivation of results and appear reasonable.

Most other investigations consider the passing rate, $\mu$, 
as an input to the model that has to be exogenously determined [see for example Gordon and Newell (1964), Brill (1972), Morse and Yaffe (1971)]. Attempts to express it as a function of the opposing traffic and passing sight distance conditions have had limited success, yielding approximate solutions for light traffic only, Gustavsson (1967).

In Appendix 1 the passing rate, $\mu$, is determined as a function of the opposing flow and the passing sight distance, with an approach used in the study of vehicular delay at unsignalized intersections [see Yeo and Weesakul (1964)]. This method takes into account the fact overlooked by others that passing occurs in bunches, when several followers take advantage of the same gap, and results are easily obtained for all levels of flow.

The following nomenclature is used in this paper:

Time process The vehicle arrival process observed by a stationary observer (headway).

Space process The process observed by an infinitely fast observer (spacing).

Leader A vehicle that is traveling unobstructed by others and at its desired speed.

Follower A non-leader vehicle.

$\mu$ Average passing rate.

$q$ Flow (vehicles/hr.).

$k$ Density (vehicles/mile).

$v$ Speed of the slow vehicles.

$V$ Desired speed of the fast vehicles.

The subindices $f f, f$ and $s$ stand for unconstrained fast, fast, and slow, respectively, e.g., $q_{f f}$ is the flow of unconstrained fast vehicles.

\subsection{Platoon length}

If traffic is very light, one can neglect the physical dimensions of the platoons (vehicles are considered mathematical points). Then to an observer moving about the stream with speed $v$, the system would look like a series of $M / M / 1$ queues with infinite capacity where the slow vehicles are the servers and the fast ones the customers.

The arrival rate, $\lambda$, to a moving queue, will be:

$$
\lambda=k_{f f}(V-v)=q_{f f}\left(\frac{V-v}{V}\right) \text {. }
$$

Jackson (1957) has shown that the queue lengths will be mutually independent geometrically distributed random variables with parameter $\rho=\lambda / \mu$. If we then let $z$, be the number of vehicles in a platoon headed by a slow vehicle (including it) we have:

$$
\begin{aligned}
\operatorname{Pr}[z=i] & =(1-\rho) \rho^{i-1} ; \quad i \geqslant 1 \\
E(z) & =(1-\rho)^{-1}
\end{aligned}
$$

where $\rho=\lambda / \mu=q_{f f} \frac{V-v}{V \mu}=q_{f f} A$.

In most practical situations one would know $q, q_{s}$ (the demand of slow vehicles), $v, V$ and only $q_{f f}$ or $k_{f f}$ would need to be known in order to determine the bunch size probabilities.
We obtain $q_{f f}$ by writing the flow of fast vehicles as the sum of the flow of constrained and unconstrained fast vehicles.

$$
\begin{aligned}
q_{f} & =q_{f f}+q_{s} E(z-1) \\
& =q_{f f}+q_{s} \frac{\rho}{1-\rho}=q_{f f}+q_{s} \frac{q_{f f}}{1-q_{f f} A} .
\end{aligned}
$$

This is a quadratic equation in $q_{f f}$ yielding:

$$
q_{f f}=\frac{1+A q-\sqrt{(1+A q)^{2}-4 A q_{f}}}{2 A}
$$

It is easy to check that $\rho$ is always less than one and that therefore a stationary solution to the idealized process always exists.

Gordon and Newell (1964) obtained $k_{f f}$ in terms of the space process ( $k$ and $k_{f}$ ), but a solution in terms of demand (flow) seems more useful.

We now let $w$, be the number of vehicles in a platoon where the unconstrained fast vehicles are regarded as one-car platoons.

The probability distribution for $w$ is written by taking into account the relative flows of unconstrained fast vehicles and slow vehicles. For the time process we get:

$$
\begin{gathered}
\operatorname{Pr}[w=1]=\frac{q_{f f}}{q_{s}+q_{f f}}+\frac{q_{s}}{q_{s}+q_{f f}}(1-\rho) \\
\operatorname{Pr}[w=i]=\frac{q_{s}}{q_{s}+q_{f f}}(1-\rho) \rho^{i-1} ; \quad i \geqslant 2 \\
E(w)=\frac{q_{f f}}{q_{s}+q_{f f}}+\frac{q_{s}(1-\rho)^{-1}}{q_{s}+q_{f f}}=\frac{q}{q_{s}+q_{f f}} .
\end{gathered}
$$

The same formulas with the $q$ 's replaced by $k$ 's apply for the space process. It must be noted that although $z$ is identically distributed for both the time and space processes, $w$ is not because a larger fraction of the unconstrained fast vehicles is observed in the time process. Formulas (5) and (6) and their counterparts for the space process are easy to use and yield the desired platoon length information. Dunne, Rothery and Potts (1968) tested a similar distribution as a descriptor of platoon length and obtained good fits with observed data.

\subsection{Delays}

It is now easy to obtain the time averaged speed of a fast vehicle.

The time going fast between queues is on the average:

$$
t_{f}=\left[k_{s}(V-v)\right]^{-1}=\left[q_{s}\left(\frac{V-v}{v}\right)\right]^{-1}
$$

The time going slow trailing in a queue is on the average:

$$
t_{s}=\frac{E(z-1)}{\lambda}=\frac{1}{\mu-\lambda}=\left[\mu-q_{f f} \frac{V-v}{V}\right]^{-1} .
$$

The total distance traveled during $t_{f}+t_{s}$ is:

$$
d=V t_{f}+v t_{s}
$$


The average speed of fast vehicles is then:

$$
\bar{u}_{f}=\frac{d}{t_{f}+t_{s}}
$$

which after some manipulations using expression (3) reduces to:

$$
\bar{u}_{f}=\frac{v}{1-\frac{q_{f f}}{q_{f}}\left(\frac{V-v}{V}\right)}
$$

Had we used the space process we would have obtained Gordon and Newell's (1964) result:

$$
\bar{u}_{f}=\frac{k_{f f}}{k_{f}}(V-v)+v
$$

It is interesting to compare (7) with Erlander's (1967) results. If one lets $V \rightarrow \infty$, then we would have obtained:

$$
\begin{aligned}
\tilde{u}_{f} & =\frac{v t_{s}+V t_{f}}{t_{s}}=v+\frac{1}{t_{s} k_{s}} \\
& =v+\frac{1}{k_{s} E \text { (waiting time) }} .
\end{aligned}
$$

This result is also obtained with Erlander's approach, but there the expected waiting time doesn't include queueing.

\subsection{The space mean speed and the fundamental diagram}

With this model one can also predict the space mean speed, $\bar{u}_{s}$, and the traffic flow density, $k$.

From the definition of space mean speed:

$$
\bar{u}_{s}=\frac{k_{f t} V+\left(k-k_{f f}\right) v}{k}=v+\frac{k_{f f}}{k}(V-v) .
$$

But (9) can also be expressed as a function of flows:

$$
\bar{u}_{s}=v+\frac{q_{f t}}{V} \frac{\bar{u}_{s}}{q}(V-v)
$$

and solving for $\bar{u}_{s}$ :

$$
\bar{u}_{s}=v\left(1-\frac{q_{f f}}{q} \frac{V-v}{V}\right)^{-1}
$$

The traffic density can also be obtained from (10) by use of $q=k \bar{u}_{s}$ :

$$
k=\frac{1}{v}\left[q-q_{f f} \frac{V-v}{V}\right]
$$

It would now be possible to predict the shape of the fundamental diagram of traffic without actual observations.

\subsection{The headway process}

We will now state two theorems, whose proofs can be found in Daganzo (1975), regarding the structure of the headway process.
Theorem 1. If we let: $X_{n}=0$ if the $n$th vehicle is a slow-leader; $X_{n}=1$ if the $n$th vehicle is a fast-follower; $X_{n}=2$ if the $n$th vehicle is a fast-leader; and denote by $Y_{n}$ the headway in front of the $n$th vehicle, then $\left[X_{n} ; Y_{n}\right]$ is a Markov-Renewal process with $S-M$ matrix:

$$
\begin{aligned}
& \mathbf{P}(t)=\left[\begin{array}{ccc}
(1-\rho) p & \rho & (1-p)(1-\rho) \\
(1-\rho) p & \rho & (1-p)(1-\rho) \\
p & 0 & (1-p)
\end{array}\right] \\
& \times\left[\begin{array}{ccc}
1-\mathrm{e}^{-\left(q_{\mathrm{s}}+q_{\mathrm{p}}\right)^{t}} & 0 & 0 \\
0 & F(t) & 0 \\
0 & 0 & 1-\mathrm{e}^{-\left(q_{s}+q_{\mathrm{f}}\right)^{t}}
\end{array}\right]
\end{aligned}
$$

where

$$
p=\frac{q_{s}}{q_{s}+q_{f f}}, \quad p=q_{f f} A
$$

and $F(t)$ represents the c.d.f. of the headway in front of a follower which, in this section, because of neglectable headways, is given by the Heavyside unit step function.

An analogous result is true for the space process.

Theorem 2. If we now redefine $X_{n}$ by letting: $X_{n}=0$ if the $n$th vehicle is a leader: $X_{n}=1$ if the $n$th vehicle is a follower; then $\left[X_{n} ; Y_{n}\right]$ is also a $M-R$ process with $S-M$ matrix given by:

$$
\mathbf{A}(t)=\left[\begin{array}{cc}
1-p \rho & p \rho \\
1-\rho & \rho
\end{array}\right]\left[\begin{array}{cc}
1-\mathrm{e}^{-\left(q_{s}+q_{t_{t} t^{t}}\right.} & 0 \\
0 & F(t)
\end{array}\right]
$$

with $p, \rho$ and $F(t)$ defined as in Theorem 1 . This is a very important result because it can be used for pedestrian delay problems, Weiss and Maradudin (1962); and with some more research for vehicular delays too. The Markovian structure of the sequence [leader-follower] has been empirically validated by Dunne, Rothery and Potts (1968).

Brill (1971) has shown that defining $X_{n}$ in the following way:

$X_{n}=0$ if $n$th vehicle is a slow or a constrained fast.

$X_{n}=1$ if $n$th vehicle is an unconstrained fast. the $\left[X_{n} ; Y_{n}\right]$ process is also $M-R$, but the $S-M$ matrix given is incorrect (Daganzo 1975).

\section{THE MEDIUM AND HEAVY TRAFFIC MODEL}

The results for light traffic were based on the two following assumptions: (i) One can observe an unconstrained fast vehicle even if a platoon is passing. This assumption is correct if gaps between platoons are very large but becomes inaccurate when flows increase. (ii) The probability of a queue being so long that it stands in the way of the next one is negligible. This assumption is the one that in the case of a unique vehicle type leads to the Borel-Tanner probabilities for platoon size. Oliver (1961) Tanner (1961a).

These two assumptions will now be relaxed and the results for light traffic generalized. For the rest of the paper we will use the following terminology:

Platoon A bunch of one or more vehicles traveling together. 
Blocking The phenomenon, associated with assumption (ii), consisting of a platoon being displaced backwards due to the spatial requirements of the previous one

Platoon interference The phenomenon, associated with assumption (i), that makes platoons longer in order to absorb the fraction of unconstrained fast vehicles that cannot be observed.

Single platoon A platoon consisting of a slow vehicle and some, if any, followers.

Composite platoon A platoon consisting of one or more single platoons running together due to the blocking phenomenon.

\subsection{Bunching}

We will first drop assumption (i) and then assumption (ii). We will also begin differentiating between the time and space processes.

Theorem 3. If we let $z_{\alpha}$ be the length of a single platoon after dropping assumption (i) and we let $F$ be the average headway in front of a follower we have:

$$
E_{s}\left(z_{a}\right)=\frac{E(z)}{1-q_{f f} F \frac{v}{V}}
$$

and

$$
E_{\mathrm{t}}\left(z_{a}\right)=\frac{E(z)}{\mathrm{I}-q_{f f} F \frac{v}{V}}\left[1+q_{f f} F \frac{V-v}{V}\right]
$$

for the space and time processes respectively.

It must be noted now that, unlike in Section 2, single platoons are longer in the time process because they include the vehicles that joined them while passing in front of the observer.

Proof. For the space process we can write:

$$
k=k_{f f}+k_{s} E(z)=k_{f f a}+k_{s} E\left(z_{a}\right)
$$

where $k_{f f a}$ is the density of unconstrained fast vehicles in the gaps between platoons. It is given by:

$$
\begin{aligned}
k_{f f a} & =k_{f f} \frac{\text { gap length-single platoon length }}{\text { gap length }} \\
& =k_{f f} \frac{k_{s}^{-1}-F v E_{s}\left(z_{a}\right)}{k_{s}^{-1}} \\
& =k_{f f}\left[1-q_{s} F E_{s}\left(z_{a}\right)\right] .
\end{aligned}
$$

Substituting into (13) and solving we obtain expression (12), for the space process.

The proof for the time process is omitted because it requires more elaboration (see Daganzo 1975).

We now relax assumption (ii). The assumption is made that if blocking occurs, the blocked platoon is displaced backwards by the blocking platoon. It is also assumed that the passing maneuvers in the blocked platoons are unaffected.
Following arguments very closely related to those leading to the Borel-Tanner distribution, the number of vehicles in a composite platoon is shown to be equivalent to the number of customers served in a busy period of a queueing system with service times given by the distribution for spacings, arrivals being compound Poisson with rate, $k_{s}$ and bunch size given by the number of vehicles in a single platoon.

This leads to a main result of the paper:

Theorem 4. Letting $z_{c}$ be number of vehicles in a composite platoon, and denoting by $N(\xi)$, its probability generating function; then, for the space process, $N(\xi)$ is given by the nontrivial solution to:

$$
N(\xi)=\frac{\xi F^{*}\left[q_{s}(1-N(\xi))\right]}{E_{s}\left(z_{a}\right)-\xi\left[E_{s}\left(z_{a}\right)-1\right] F^{*}\left[q_{s}(1-N(\xi))\right]}
$$

where $F^{*}(\cdot)$ is the Laplace transform of $F(t)$, the c.d.f. for the headway in front of a follower. (No longer a Heavyside unit step function).

A few algebraic manipulations then yield:

$$
E_{\mathrm{s}}\left(z_{c}\right)=\frac{E_{s}\left(z_{a}\right)}{1-\rho_{s}} \quad \text { with } \quad \rho_{\mathrm{s}}=q_{\mathrm{s}} F E_{\mathrm{s}}\left(z_{a}\right)<1
$$

The solution exists iff $\rho_{s}<1$, which puts a capacity bound on the amount of traffic the road can serve. It is interesting to note that Miller (1963) used a multiplier, $1 /(1-x)$ similar to the $1 /\left(1-\rho_{s}\right)$ to correct the average platoon length and that is now theoretically justified.

If we let $\gamma_{n s}^{2}=\left[\operatorname{var}_{s}\left(z_{c}\right) \mathrm{j} /\left[E_{s}^{2}\left(z_{c}\right)\right]\right.$ expression (14) also yields:

$$
y_{n s}^{2}=\frac{\rho_{s}}{1-\rho_{s}}+\frac{E_{s}\left(z_{a}\right)-1+\left(\gamma_{F} \rho_{s}\right)^{2}}{E_{s}\left(z_{a}\right)\left(1-\rho_{s}\right)}
$$

where $\gamma_{F}^{2}$ is the coefficient of variation of $F(t)$.

Similar formulae exist for the time process.

This result generalizes and brings together two different classical formulations of the problem, one of Oliver (1961) and Tanner (1961a), and that of Gordon and Newell (1964). The results of Gordon and Newell for light traffic are obtained by neglecting the physical dimensions of the vehicles $\left(F=0 ; F^{*}(s)=1\right)$. In that case, eqn (14) becomes:

$$
N(\xi)=\frac{\xi}{E_{s}\left(z_{a}\right)-\xi\left[E_{s}\left(z_{a}\right)-1\right]}
$$

but from (12) we see that $E_{s}\left(z_{a}\right)=E(z)$ and hence:

$$
N(\xi)=\frac{\xi}{E(z)-[E(z)-1] \xi}=\xi \frac{1-\rho}{1-\rho \xi}
$$

which is the generating function of the probabilities in (2).

The Borel-Tanner probabilities are also obtained as an extreme case of Theorem 4 . If $q_{f}=0$ and the headways are deterministic $\left(F^{*}(s)=\mathrm{e}^{-\mathrm{s} F}\right)$ eqn (14) becomes:

$$
N(\xi)=\xi \exp [-k F v(1-N(\xi))]
$$


which is the equation yielding the Borel-Tanner probabilities.

\subsection{Platoon length distributions:}

Equation (14) cannot be inverted but a good approximation to the composite platoon distribution with the same mean and variance is given by the following mixture of geometric distributions:

$$
\operatorname{Pr}\left[z_{c}=n\right]=\frac{\left(1-\rho_{1}\right)^{2} \rho_{1}^{n-1}+\left(1-\rho_{2}\right)^{2} \rho_{2}{ }^{n-1}}{\left(1-\rho_{1}\right)+\left(1-\rho_{2}\right)}
$$

with

$$
\begin{aligned}
& \rho_{1}=1-\frac{1}{E_{s}\left(z_{c}\right)}+\frac{1}{E_{s}\left(z_{c}\right)} \sqrt{1-\frac{2 E_{s}\left(z_{c}\right)}{1+E_{s}\left(z_{c}\right)\left(1+\gamma_{n s}^{2}\right)}} \\
& \rho_{2}=1-\frac{1}{E_{s}\left(z_{c}\right)}-\frac{1}{E_{s}\left(z_{c}\right)} \sqrt{1-\frac{2 E_{s}\left(z_{c}\right)}{1+E_{s}\left(z_{c}\right)\left(1+\gamma_{n s}^{2}\right)}}
\end{aligned}
$$

Analogous formulas trave been derived for the time process. We now want to determine the platoon length distribution (inciuding unconstrained fast vehicles).

Letting $w_{c}$ be the number of vehicles in a platoon and following the same argument that led to expression (5):

$$
\begin{aligned}
& \operatorname{Pr}\left[w_{c}=1\right]=\frac{k_{f f}}{k_{s}+k_{f f}}+\frac{k_{s}}{k_{s}+k_{f f}} \operatorname{Pr}\left[z_{\mathrm{c}}=1\right] \\
& \operatorname{Pr}\left[w_{c}=i\right]=\frac{k_{s}}{k_{s}+k_{f f}} \operatorname{Pr}\left[z_{c}=i\right] ; \quad i \geqslant 2 .
\end{aligned}
$$

For the mean and variance of $w_{c}$ we obtain:

$$
\begin{gathered}
E\left(w_{c}\right)=\frac{k_{f f}+k_{s} E_{s}\left(z_{c}\right)}{k_{f f}+k_{s}} \\
\operatorname{var}\left(w_{c}\right)=\frac{k_{s}}{k_{s}+k_{f f}}\left[\frac{k_{f f}}{k_{s}+k_{f f}}\left[E_{s}\left(z_{c}\right)-1\right]^{2}+\gamma_{n s}^{2} E_{s}^{2}\left(z_{c}\right)\right] .
\end{gathered}
$$

Similar expressions hold for the time process.

\subsection{Delays and the fundamental diagram}

It can be shown, Daganzo (1975), that as long as $\rho_{s}<1$, the formulas derived in Sections 2.2 and 2.3 still hold after dropping assumptions (i) and (ii). Hence it is valid to determine the fundamental diagram and evaluate delays with the formulas for light traffic.

\subsection{The headway and spacing process}

After the blocking phenomenon is introduced, we must give up the Markov-Renewal property of the spacing process. However, if we are ready to accept formula (17) for the number of vehicles in a composite platoon, a 4-state Markov-Renewal structure can be justified. The $S-M$ matrix can be found in Daganzo (1975).

It is of interest to note that the headway process follows the same behavior as the spacing process and that a coarser approximation by a 2 -state $M-R$ process can also be made in both cases.

This coarser approximation was used to predict the lag-one autocorrelations of an observed headway stream and the results matched fairly well with the observed correlations (Daganzo 1975).

\section{AN EXAMPLE}

For illustrative purposes, we will derive the fundamental diagram and the platoon length distribution for a hypothetical case where:

$$
V=60 \mathrm{MPH} ; \quad v=30 \mathrm{MPH} ; \quad q_{s}=0.1 q \mathrm{VPH} .
$$

We will also assume that the passing rate $\mu$ is given by:

$$
\mu=637 \exp \left(-\frac{q}{153}\right) \text { (passings per hour). }
$$

Assuming that the traffic in the other direction is approximately equal to the traffic in our direction, the above exemplifies how $\mu$ diminishes with increasing $q$, according to Appendix 1.

It has been mentioned that expression (10) is good for all ranges of $q$ as long as $\rho_{\mathrm{s}}<1$. Hence:

$$
\begin{aligned}
& \tilde{u}_{s}=\frac{v}{1-\frac{q_{f f}}{q}\left(\frac{V-v}{V}\right)} \text { with } \\
& q_{f f}=\frac{1+A q-\sqrt{(1+A q)^{2}-4 A q_{t}}}{2 A}
\end{aligned}
$$

and

$$
A=\frac{V-t}{V_{\mu}}
$$

Direct substitution of our data with different values of $q$ yields the results displayed in Fig. 1.

Note the sudden drop in the space mean speed when the flow reaches about $300 \mathrm{VPH}$. This sudden drop or "phase change" was also reported by Morse and Yaffe (1971) and is typical of two-lane roads.

We will now derive the platoon length distribution for the space process for a flow level of $800 \mathrm{VPH}$ and assuming that $F=2.5$ secs., and $\gamma_{F}^{2}=0$.

We will make use of formulas (18) and (17) to determine the distribution. These formulas require the use of expressions (16), (15), (12), (4) and (2).

From the data: $\quad q_{f}=720 \mathrm{VPH}$

$$
\mu=2.5 \text { PPM } \approx 0
$$

expression (4) yields $q_{f f}=4 \cdot 5 \approx 0$ and from (2), $E(z)=10$. We now take into account the blocking phenomenon by computing $E_{s}\left(z_{c}\right)$ and $\gamma_{n s}^{2}$ using (15) (16):

$$
\begin{aligned}
E_{s}\left(z_{c}\right) & =22.5 \\
\gamma_{n s}^{2} & =3.28 .
\end{aligned}
$$

Note that now that the light traffic hypotheses have been dropped, platoons are notably longer and more unpredictable. 


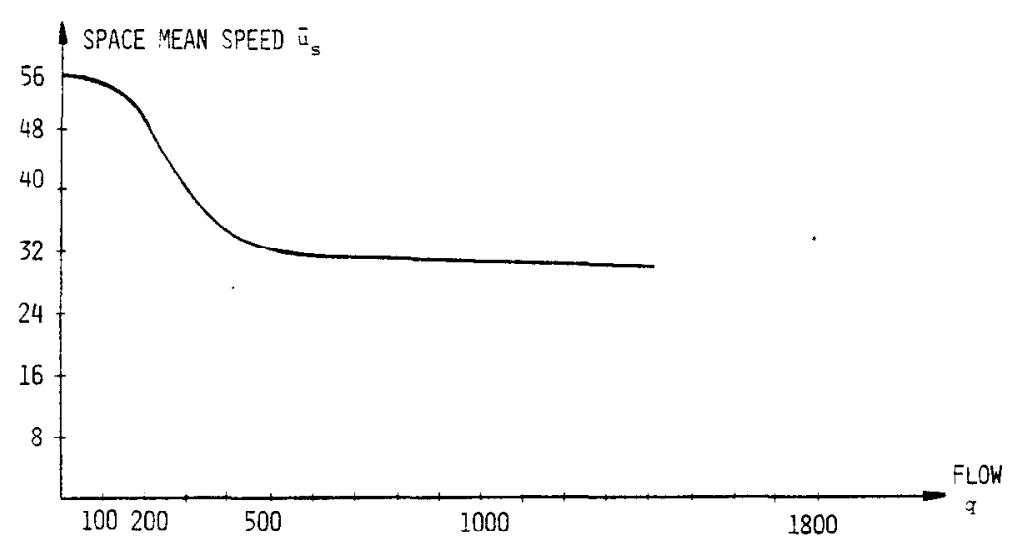

Fig. 1. A flow-speed diagram.

The composite platoon length is given by (17) with:

$$
\rho_{1}=0.988 ; \quad \rho_{2}=0.923 \text {. }
$$

Substitution into (18) would yield the sought probabilities. The method is easy for both manual and automatic computation and the results obtained should be no worse than the geometric or the Borel-Tanner distributions, since the model generalizes both. (see Daganzo 1975).

\section{THE NO-PASSING CASE}

In Fig. 1 we see that except for very light traffic, passing is impossible and because of that, the space mean speed drops toward the minimum value very rapidly.

In many practical situations, the levels of flow will be above the critical level and no great mistake will be made by considering no-passing conditions.

The no-passing assumption simplifies all the results of the paper and they are summarized below:

\subsection{Platoon length}

Taking limits on (4) as $A \rightarrow \infty$

$$
\rho=q_{f f} A=\frac{q_{f}}{q} \text { and } q_{g}=0 .
$$

From expressions (12), (6) and (2):

$$
E_{i}\left(z_{a}\right)=E_{s}\left(z_{a}\right)=E(z)=E(w)=\frac{1}{1-\rho}=\frac{q}{q_{s}} .
$$
(15):

and

$$
\rho_{s}=q F
$$

$$
E_{s}\left(z_{c}\right)=E_{t}\left(z_{c}\right)=\frac{q}{q_{s}(1-q F)} .
$$

The coefficient of variation is:

$$
\gamma_{u s}^{2}=\gamma_{n t}^{2}=\frac{q F+q_{f} / q+\left(\gamma_{F} q F\right)^{2}}{1-q F} .
$$

\subsection{The headway process}

The $S-M$ matrix given is theorem 2 becomes:

$$
A(t)=\left[\begin{array}{ll}
1-\rho & \rho \\
1-\rho & \rho
\end{array}\right]\left[\begin{array}{cc}
1-\mathrm{e}^{-q_{\mathrm{s}} t} & 0 \\
0 & F(t)
\end{array}\right]
$$

which corresponds to a degenerated $M-R$ process. The process is renewal, as assumed in the classical theory of traffic, with headway distribution given by:

$$
\operatorname{Pr}\left[Y_{n} \leqslant t\right]=\frac{1}{q}\left[q_{f} F(t)+q_{s}\left[1-\mathrm{e}^{-q_{s} t}\right]\right]
$$

The renewal property of the headway (spacing) process is only approximate for the heavy traffic case, but a formula similar to (27) has also been derived (Daganzo 1975).

\section{DISCUSSIUN}

A predictive model for traffic in a two-lane bidirectional road has been presented. Similar models in the literature always assume that vehicles have no physical dimensions and that a platoon cannot be long enough to "block" the progression of the next one and this severely restricts their applicability to light traffic and decreases their usefulness. By allowing vehicles to have physical dimensions and travel at two possible speeds, a hybrid model of Oliver-Tanner and Gordon-Newell has been developed.

The passing rate, $\mu$, is derived in the Appendix following an approach similar to Yeo and Weesakul's vehicular delay at unsignalized intersections which adds to the realism of the model.

Dunne et al. (1968) validated the Markovian structure of the [leader-follower] process and Daganzo (1975) showed that the presented method significantly improves the platoon length predictions of Oliver-Tanner's and Gordon-Newell's.

Since the model also yields information on capacity, delays and the fundamental diagram, it can be a valuable tool to evaluate the effects of such things as: banning trucks and slow vehicles on certain occasions, improving sight distance conditions, lowering speed limits, and estimating delays to crossing streams of pedestrians and vehicles. 
The model could also be used to predict the level of head-on collisions on a section of the road and to compare different situations before the accidents do happen. The measure of performance could be the number of passings per unit time in that section of the road times the opposing flow $\left[q_{s} q_{f f} q_{0}(V-v) /(V v) L\right)$. (For the example of Section 4 , the critical flow is $q=350 \mathrm{VPH}$; smaller flows have less conflicts, and larger flows provide less opportunities to pass).

The author feels that the constant above described is strongly correlated with the accident liability of different locations and situations. Once enough data is available, an econometric model might be developed to predict the absolute number of head-on collisions in two lane roads.

Further work related to the topic presented in the paper should focus in developing methods for the estimation of рагаmeters [see Daganzo (1975) for an ad-hoc method, and some recommendations in this respect], and eventually integrate it into studies of pedestrian delay and vehicular delay by developing tables and/or a set of computer programs.

Acknowledgement-I greatly appreciate all the criticisms and suggestions of Dr. D. E. Cleveland from the University of Michigan whose review of the paper very much influenced its final form.

\section{REFERENCES}

Andrews F. C. (1970) A statistical theory of traffic flow on highways. I-steady state flow in low density limit II-three car interactions and the onset of queueing. Trans. Res. 8, 359-365.

Brill E. A. (1971) Point processes arising in vehicular traffic flow J. Appl. Prob. 8. 809-814.

Bullen A. G. R. and Worrall R. D. (1970) Formulating a model of multilane traffic flow. Highway Res. Rec. 334, 34-38.

Carleson L. (1957) Eu Matematisk Modell for Landsvagstrafik Nord. Mathematisk Tidskrift 5, 175-180.

Çinlar E. (1969) Markov renewal theory. Adv. Appl. Prob. 1(2), 123-187.

Cox D. R. and Smith W. L. (1961) Queues. Chapman and Hall, London.

Daganzo C. F. (1975) Two-land road traffic, A stochastic mode Ph.D. Thesis. The University of Michigan.

Disney R. L. and Cherry W. P. (1973) Some Topics in Queueing Network Theory. Tech-Rep. 73-4. Department of Industrial and Operations Engineering, The University of Michigan.

Dunne M. C., Rothery R. W. and Potts R. B. (1968) A discrete Markov model of vchicular traffic. Transpn. Sci. 2, 233-251.

Erlander S. (1967) A Mathematical Model for Traffic on a Two-Lane Road. Vehicular Traffic Science (Edited by L. C. Edie, et al.) pp. 153-167. American Elsevier, New York.

Erlander S. (1971a) A mathematical model for traffic on a two-lane road with some empirical results-I. Theoretical model and estimation problems. Transpn. Res. 5, 135-i47.

Erlander S. (1971b) A mathematical model for traffic on a two-lane road with some empirical results-II. Empirical results Transpn. Res. 5. 149-175.

Galin D. and Epstein B. (1974) Speeds and delays on two-lane roads, where passing is possible at given points of the road. Transpn. Res. 8. 29-37.

Gordon W. J. and Newell G. F. (1964) Equilibrium analysis of a stochastic model of traffic flow. Pro. Cam. Philo. Soc. 60 227-236.

Gustavsson J. (1967) A model for overtaking on a two-lane road with limited visibility. Vehicular Traffic Science (Edited by L. C. Edie, et al.) pp. 138-152.

Haight F. A. (1963) Mathematical Theories of Traffic Flow Academic Press. New York.
Jackson T. R. (1957) Networks of waiting lines. Ops. Res. 5, 518-521.

Jewell W. S. (1965) Markov-Renewal models in traffic flow theory. Proc. 3rd. Int. Symp. Theory of Traffic Flow. New York.

Kemeny J. and Snell L. (1959) Finite Markov Chains. D. Van Nostrand, Inc.

Miller A. J. (1961) A queueing model for road traffic flow. J. Roy Stat. Soc. B23, 64-75.

Miller A. J. (1962) Road traffic flow considered as a stochastic process. Pro. Cam. Philo. Soc. 58, 312-325

Miller A. J. (1963) Analysis of bunching in rural two-lane traffic Ops. Res. 11, 236-247.

Miller A. J. (1967) Queueing in rural traffic. Vehicular Traffic Science. (Edited by L. C. Edie, et al.) ppp. 127-137. American Elsevier, New York.

Morse P. M. and Yaffe H. J. (1971) A queueing model for car passing. Transpn. Sci. 5, 48-63.

Newell G. F. (1955) Mathematical models for freely flowing highway traffic. J. Oper. Res. Soc. Am. 3, 176-186.

Newell G. F. (1966) Equilibrium probability distribution for low density highway traffic. J. Appl. Prob. 3, 247-260.

Oliver R. M. (1961) A traffic counting distribution. Oper. Res. 9, 802-810.

Tanner J. C. (1961a) A derivation of the Borel distribution Biometrika 48, 222-224.

Tanner J. C. (1961b) Delays on a two-lane road. J. Stat. Soc. B23. 38-63

Weiss G. H. and Maradudin A. A. (1962) Some problems in traffic delay. Ops. Res. 10, 74-104.

Yeo G. F. and Weesakul B. (1964) Delays to road traffic at an intersection. J. Appl. Prob. 1, 297-310.

Yeo G. F. (1964) Traffic delays on a two-lane road. Biometrika 51 11-15.

\section{APPENDIX 1}

The passing constant as a function of flow and passing conditions

In all the derivations of the paper, it has been assumed that the passing rate, $\mu$, is known and possibly exogenously determined. It is very convenient to express such a constant as a function of the opposing flow and the passing conditions of the roadway because, then, the predictive capabilities of the model are greatly improved.

As in the vehicular delay problem at an unsignalized intersection, the trailing vehicles in a platoon have two types of service times: those of the customers that find nobody queueing at their arrival time, and those that have to queue; see for example Yeo and Weesakul (1964).

The influence of the roadway is approximately accounted for with a single constant, $q_{e}$, that is taken to be the equivalent amount of traffic on a road with perfect sight distance.

The critical gap, $T$, is taken to be deterministic within and across the population and traffic in the opposing lane Poisson with rate $q_{0}$.

We will further assume that the speeds in the opposing lane are similar to those in the studied lane and hence that the rate of equivalent traffic observed by a following vehicle is approximately:

$$
2\left(q_{e}+q_{0}\right)
$$

This assumption can easily be generalized but we do not believe that it is necessary.

We now proceed and derive the mean service times of queueing and non-queueing customers. The delay to a 
non-queueing customer, except for a reaction time $F_{f}$, will be on the average approximately:

$$
\bar{t}_{0}=\frac{\mathrm{e}^{2\left(q_{e}+q_{0 j}\right) T}-2\left(q_{e}+q_{0}\right) T-1}{2\left(q_{e}+q_{0}\right)}
$$

as given by the asynchronous pedestrian delay, Tanner (1961b).

The service time of a queueing customer will be derived subsequently:

With probability $e^{-2\left(q_{e}+q_{0}\right) F_{f}}$ nobody arrives in the opposite lane during the move-up time, $F_{f}$, and the service time is equal to the move-up time. With probability, $1-\mathrm{e}^{-2\left(a_{e}+q_{0}\right) F_{l}}$, during its move-up time some vehicle arrives in the opposite lane and the service time will be longer than $T$ so that in total:

$$
t_{1}=\mathrm{e}^{-2\left(u_{e}-u_{0}\right) F_{f}} F_{f}+\left(1-\mathrm{e}^{-2\left(u_{\mathrm{e}}+u_{\mathrm{g}}\right) F_{f}}\right)\left(T+Y+t_{0}\right)
$$

where $t_{0}$ is the synchronous or asynchronous pedestrian delay in Poisson traffic, and $T+Y$ is the time until the first arrival in the opposite land that we know happens in the interval $\left[T, T+F_{f}\right]$ hence: $0 \leqslant Y<F_{f}$.

Momentarily let:

$$
q=2\left(q_{e}+q_{0}\right)
$$

then

$$
\begin{aligned}
P_{r}[Y \leqslant y] & =\frac{q \mathrm{e}^{-q y}}{1-\mathrm{e}^{-q F_{j}}}, \quad 0 \leqslant Y<F_{f} \\
& =0 ; \quad \text { otherwise }
\end{aligned}
$$

and

$$
E(y)=\frac{1}{q}-\frac{F_{f}}{\mathrm{e}^{q F_{f}}-1} .
$$

Taking expectations on eqn (29) and substituting, we get:

$$
\bar{t}_{1}=\left(1-\mathrm{e}^{-q F_{r}}\right) \frac{\mathrm{e}^{4 T}}{q}=\left(1-\mathrm{e}^{-3\left(q_{e}+q_{1}\right) F_{r}}\right) \frac{\mathrm{e}^{2\left(q_{e}+q_{0}\right) T}}{2\left(q_{e}+q_{0}\right)}
$$

as the average service time for those vehicles that have to queue.

With the type of queueing system considered here, the average number of followers in one queue, $E(z-1)$, is given by:

$$
E(z-1)=\frac{\left(\lambda \bar{t}_{1}\right)^{2}}{1-\lambda \bar{t}_{1}}+\frac{\lambda \bar{t}_{2}+\left(\lambda \bar{t}_{2}\right)^{2}-\left(\lambda \bar{t}_{1}\right)^{2}}{1-\lambda \bar{t}_{1}+\lambda \bar{t}_{2}}
$$

where $\bar{t}_{2}=\bar{t}_{0}+F_{f}$ and $\lambda=q_{f f} \frac{V-v}{V}$

(for simplicity we assume that $t_{0}$ and $t_{1}$ have unit coefficients of variation).

Following the same arguments of the text, we rewrite eqn (3):

$$
q_{f}=q_{f f}+q_{s} E(z-1)
$$

which, combined with (31) allows us to get $q_{f f}$ and $E(z)$ iteratively.

The equivalent passing rate for negative exponential service times is obtained by setting:

$$
E(z)=\left(1-q_{f} \frac{V-v}{V \mu}\right)^{-1}
$$

with the values obtained above.

This method of computing $q_{f f}, E(z)$ and $\mu$ is very easily programmable and was used for the field testing of the model. 